\title{
Proyecto de mejora del índice de satisfacción del cliente en el proceso de Animación en el hotel Meliá Las Antillas de Varadero
}

DOI: $\underline{\text { https://doi.org/10.33262/ap.v1i1.16 }}$

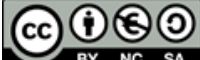

Project to improve the customer satisfaction index in the Animation process at the Meliá Las Antillas hotel in Varadero

Bisleivys Jiménez Valero., ${ }^{1}$, Mabielys Lorenzo Puentes. ${ }^{2} \&$ Lisdeybis Pino Acosta. ${ }^{3}$

\begin{abstract}
.
The research is carried out in the Animation area of the "Meliá Las Antillas" hotel located on the Varadero peninsula in the province of Matanzas. It addresses the issue of customer satisfaction, where despite working to obtain high satisfaction rates, a high number of customer complaints are reflected, which constitutes a scientific problem to be solved. The general objective is to propose a project to improve the customer satisfaction index in the Hotel Animation process. To do this, a procedure is followed that integrates project management techniques in their identification and planning phases, as well as interviews with staff, document review and direct observation. As a result, two alternative solutions to the identified problem are obtained, however, one of them is more viable than the other, which is proposed to the entity for its execution and control.

${ }^{1}$ Doctora en Ciencias Técnicas. Máster en Gestión Turística con Mención en Gestión Hotelera. Ingeniera Industrial. Profesora Titular del Departamento de Turismo. Cuba. Teléfono: (045) 256889. bisleivys.jimenez@umcc.cu

${ }^{2}$ Estudiante de la carrera de Turismo. Universidad de Matanzas. Cuba. mabielys.lorenzo@est.umcc.cu

${ }^{3}$ Estudiante de la carrera de Turismo. Universidad de Matanzas. Cuba. lisdeybis.pino@est.umcc.cu
\end{abstract}


Keywords: hotel animation, tourism management, customer satisfaction, tourist quality, animation project.

\section{Resumen.}

La investigación se realiza en el área de Animación del hotel "Meliá Las Antillas" ubicado en la península de Varadero en la provincia de Matanzas. Aborda el tema de la satisfacción del cliente, donde a pesar de que se trabaja por obtener altos índices de satisfacción se refleja un alto número de quejas por parte de los clientes, lo que constituye un problema científico a resolver. El objetivo general consiste en proponer un proyecto para la mejora del índice de satisfacción del cliente en el proceso de Animación del hotel. Para ello se sigue un procedimiento que integra las técnicas de gestión de proyectos en sus fases de identificación y planificación, así como entrevistas con el personal, revisión de documentos y observación directa. Como resultado se obtienen dos alternativas de solución a la problemática identificada, no obstante, una de ellas es más viable que la otra, la cual se le propone a la entidad para su ejecución y control.

Palabras claves: animación hotelera, gestión turística, satisfacción del cliente, calidad turística, proyecto de animación.

\section{Introducción.}

El turismo comprende el conjunto de actividades que realizan las personas durante sus viajes y estancias, en lugares distintos al de su entorno habitual por un período de tiempo consecutivo inferior a un año, con fines de ocio, por negocios y otros motivos, no relacionados con el ejercicio de una actividad remunerada en el lugar visitado (OMT, 2003). Es considerado uno de los sectores más dinámicos a nivel mundial y es comúnmente denominado la locomotora económica de los países, incluso, llega a ser en muchos casos, su primer renglón en ingresos (Padilla Rodríguez, 2015).

Un número creciente de destinos de todo el mundo se han abierto al turismo y han invertido en él, haciendo del mismo un sector clave para el progreso socioeconómico, a través de la creación de puestos de trabajo y de empresas, la generación de ingresos de exportación y la ejecución de infraestructuras (OMT, 2015).

Durante las seis últimas décadas, el turismo ha experimentado una continua expansión y diversificación, convirtiéndose en uno de los sectores económicos de mayor envergadura y crecimiento del mundo (OMT, 2015).

Varadero garantiza para todos, excelentes ofertas de alojamiento en instalaciones de varias estrellas, entre villas, bungalows, hoteles y aparthoteles, situados en primera línea de playa o en avenidas aledañas. La red hotelera ha crecido considerablemente en los últimos años y continúa a un acelerado ritmo, por la constante demanda de turistas que seleccionan a Varadero para unas inolvidables vacaciones. 
El hotel Meliá Las Antillas dedicado al segmento de personas mayores de 16 años tiene como objetivo fundamental cumplir con lo establecido en el lineamiento 235 de la actual política económica y social de Cuba el cual plantea la necesidad de ingresar divisas frescas al país. En correspondencia con esto, la instalación se ha trazado entre sus líneas de trabajo la creación de nuevas ofertas competitivas en el mercado que sean del agrado de los clientes y que satisfagan sus más diversas expectativas en aras de incrementar su imagen y situarse en la preferencia de los clientes en el destino.

La satisfacción del cliente externo es uno de los eslabones fundamentales para el afianzamiento del producto hotelero en el mercado puesto que el hotel se caracteriza por el servicio personalizado que les brinda a sus clientes. De ahí la necesidad de crear novedosas propuestas que garanticen la satisfacción de los clientes para evitar la pérdida de mercados potenciales para lo cual se plantea como situación problémica la siguiente:

El hotel a pesar de contar con un equipo de animación altamente calificado y que presta sus servicios durante todo el día y parte de la noche cumpliendo con un amplio programa de recreación diseñado para el disfrute de los clientes, que incluyen actividades deportivas, recreativas y culturales ha presentado varios problemas en lo referido a la calidad de la prestación del servicio en el proceso de animación:

- Carencia de actividades nocturnas para personas de la tercera edad en horarios que se ajusten a las características y necesidades las mismas. La mayoría de estas tienen lugar muy tarde en la noche.

- Poco entusiasmo por parte de los animadores.

- Repetividad en la animación y poca variedad de la misma. Los shows se centran demasiado en el baile y no explotan otras variantes artísticas.

- Falta de actividades recreativas en el área de la playa.

- Pocos establecimientos para el ocio y la recreación.

- Necesidad de una mayor personalización en las actividades recreativas y de los animadores.

- Impuntualidad en las actividades recreativas planificadas.

- Insuficientes sillas para todos los huéspedes en las áreas recreativas.

- Se pone poca música cubana.

- Los animadores no hacen partícipes a los huéspedes.

- Poca variedad en el show.

- Los espectáculos no son de buen gusto, el audio es muy ruidoso.

- La música en la playa está muy alta.

- Falta animación en alemán.

- Pocas actividades de bailes con los clientes después del show alrededor de la piscina.

Analizando lo anteriormente expuesto se define como problema científico: 
¿Cómo desarrollar actividades recreativas y de animación que satisfagan las exigencias y expectativas del cliente que visita la entidad teniendo en cuenta que el mercado al que está dirigido es para mayores de 16 años?

En correspondencia con los aspectos señalados anteriormente, el objetivo general consiste en: elaborar un proyecto cuya finalidad es mejorar el índice de satisfacción del cliente en el proceso de animación.

Para dar cumplimiento al objetivo trazado se utilizan técnicas y herramientas tales como: los árboles de problemas y el de objetivos, la matriz de marco lógico, los diagramas de redes y el de PERT, la matriz de responsabilidades, la estructura fraccionada del trabajo, entre otras.

\section{Desarrollo.}

El hotel Meliá Las Antillas, perteneciente al estado cubano y a la cadena hotelera Cubanacán, se encuentra ubicado en la primera línea de playa en el Sector de los Taínos, a unos $5 \mathrm{~km}$. del centro histórico de la ciudad varaderense. Inició sus operaciones en el año 1999 bajo administración mixta con la compañía Sandals de Jamaica y en el año 2006 bajo contrato de administración con la cadena Sol Meliá.

Es una instalación de categoría de 4 estrellas Plus y funciona bajo la modalidad de "Todo Incluido". Tiene un total de 350 habitaciones todas Junior Suite distribuidas de la manera siguiente: 42 económicas, 238 estándar, 68 superiores y 2 Suites, rodeadas de una exuberante vegetación, hermosos jardines y espejos de agua.

El producto Meliá Las Antillas se encuentra diseñado para parejas, familias, y grupos de jóvenes con edades superiores a 16 años. Los mercados que aparecen con mayor representatividad son: Canadá, Alemania, Francia, Rusia y Holanda.

El hotel cuenta con un equipo de animación que presta sus servicios durante todo el día y parte de la noche, cumpliendo con un amplio programa de recreación diseñado para el disfrute de los clientes, que incluyen actividades deportivas, recreativas y culturales, todo esto incluido en los programas de animación, diurno, nocturno, y emergente, avalados por la dirección del departamento y del hotel.

El departamento cuenta con todo el equipamiento necesario para sus actividades, con excepción de algunas dificultades con los equipos de audio.

El $80 \%$ de la música que se escucha en el hotel es cubana, se realizan clases de coctelería cubana, baile y del idioma español.

Para la realización de estas actividades se cuenta con una plantilla de animación compuesta por 18 trabajadores, los cuales comienzan sus labores en horario diurno a las 9 am con una reunión de apertura para la distribución de las actividades del día y la noche según el programa de animación. Solamente entran a las $5 \mathrm{pm}$ el presentador de la noche y un jefe de actividades, o sea, de 5pm a 1 am para concluir en la discoteca. Todos los animadores tienen presentación una vez a la semana y a su vez deben recibir a los clientes en el bufet.

A continuación, se exponen los resultados de la aplicación de las herramientas de gestión de proyectos: 


\section{Análisis de la situación.}

\section{Situación actual:}

- Hotel perteneciente a la cadena hotelera CUBANACÁN y contrato de administración con la cadena Española Sol Meliá.

- Ubicado en la primera línea de playa en el sector de "Los Taínos" y a 5 km. Del centro histórico de Varadero.

- Tiene categoría cuatro estrellas y opera bajo la modalidad todo incluido.

- Posee un total de 350 habitaciones, rodeadas de una exuberante vegetación, hermosos jardines y espejos de agua.

- Producto diseñado para parejas, familias y grupo de jóvenes mayores de 16 años.

- Entre los principales mercados emisores se destacan: Canadá, Alemania, Francia, Rusia y Holanda.

- Cuenta con 5 bares y 3 restaurantes especializados.

- Posee un equipo de animación durante todo el día y parte de la noche, con un amplio programa de animación que incluye actividades deportivas, recreativas y culturales.

- El departamento de animación cuenta con los equipos necesarios para las actividades, con excepción de algunas dificultades con el audio.

- El $80 \%$ de la música que se escucha es cubana y se realizan clases de coctelería, baile y del idioma español.

- Posee un total de 366 trabajadores para la realización de las actividades y una plantilla de animación de 18 trabajadores.

- Desde finales del pasado año y hasta la fecha (Octubre- Mayo), el departamento ha sido objeto de quejas por parte de los clientes, con un total de 1198 comentarios negativos, entre ellos:

- Carencia de actividades nocturnas para personas de la tercera edad en horarios que se ajusten a las características y necesidades las mismas.

- Poco entusiasmo por parte de los animadores

- Repetividad en la animación y poca variedad de la misma.

- Falta de actividades recreativas en el área de la playa.

- Pocos establecimientos para el ocio y la recreación.

- Necesidad de una mayor personalización en las actividades recreativas y de los animadores.

- Impuntualidad en las actividades recreativas que habían sido planificadas.

- No alcanzan las sillas para todos los huéspedes en las áreas recreativas.

- Se pone poca música cubana.

- Los animadores no hacen partícipes a los huéspedes.

- Los espectáculos no son de buen gusto, el audio es muy ruidoso.

○ La música en la playa está muy alta, falta animación en alemán. 
- Debiera haber más baile con los clientes después del show, alrededor de la piscina y no en la discoteca.

En inspecciones realizadas se detectaron las deficiencias siguientes:

- No se hace el recorrido por la playa con frutas, el que pudiera servir como medio de comunicación directa con los clientes y fuente de información.

- Aunque prácticamente se cumple en el hotel lo establecido sobre el $80 \%$ de la música que debe ser cubana, esto se hace de forma deliberada, es decir, se pone la música a gusto del o los animadores y casi siempre es la misma.

- En las clases de baile, solo se enseña salsa y reggaetón.

- Existen áreas de buena calidad para la realización de algunas actividades que no se aprovechan.

- Algunas actividades de piscina como el Aqua Gim casi nunca cuentan con buena participación de los clientes.

- Los carteles con los programas de animación solo se encuentran situados en el área del lobby inferior.

\section{Situación deseada:}

- Elevada aceptación por parte del cliente del producto animación.

- Elevar el sentido de pertenencia de los trabajadores del departamento de animación.

- Personal capacitado, rápido y eficiente.

- Incrementar la frecuencia, variedad, control y exigencia al talento artístico contratado.

- Posicionar al hotel en un lugar privilegiado en la mente del cliente.

\section{Situación futura de no acometerse el proyecto:}

- Bajo índice de satisfacción del cliente.

- Pérdida de clientes y de mercado por no encontrarse el hotel dentro de sus expectativas ni por lo que pagó.

- Personal con bajo sentido de pertenencia, ineficiencia en el servicio y bajo nivel idiomático.

- Bajos ingresos por concepto de comercialización.

\section{Análisis de la Participación.}

\section{Grupos de interés para el proyecto:}

- MINTUR: Grupo rector de la actividad turística.

- CUBANACÁN S.A Casa Matriz: Organización superior. 
- SOL MELIÁ Hotels and Resorts: Cadena Hotelera española bajo contrato de administración con el grupo hotelero cubano CUBANACÁN S.A.

\section{Beneficiarios Directos:}

- Hotel MELIÁ Las Antillas

- Clientes

- TTOO

- TURARTE

\section{Beneficiarios Indirectos:}

- Trabajadores de la instalación

- MINTUR

- Proveedores de productos y equipos de animación

\section{Involucrados:}

- MINTUR

- CUBANACÁN S.A

- Hotel MELIÁ Las Antillas

- TURARTE

Para el análisis de los involucrados se realizó la matriz de involucrados mediante la cual se arribó a la conclusión de que la institución que más responsabilidad tiene en la realización de este proyecto es el propio hotel MELIÀ Las Antillas.

\section{Análisis de los Problemas}

Para la realización del árbol de problemas se realizó una tormenta de ideas detectando los principales problemas de la entidad mencionados anteriormente en el análisis de la situación concluyendo que el problema oportunidad es el bajo índice de satisfacción del cliente en el proceso de Animación.

\section{Análisis de los objetivos}

Para determinar el objetivo principal del proyecto se realizó el árbol de objetivos resultando como objetivo fundamental: elevar el índice de satisfacción del cliente en el proceso de animación.

\section{Análisis de las alternativas}

Después de analizado el objetivo fundamental del proyecto se procedió a determinar las principales alternativas; 
- Alternativa A: contratar un personal altamente calificado y con elevado sentido de pertenencia para las actividades de animación.

- Alternativa B: mejorar la profesionalidad de los animadores mediante cursos de capacitación, tanto en la comunicación idiomática como Interpersonal para mejorar la forma de interactuar con los clientes.

\section{Estudio de prefactibilidad:}

\section{Prefactibilidad de Mercado:}

- Demanda inicial probable de los principales mercados emisores: Canadá, Alemania, Francia, Rusia y Holanda.

- Creación de una mayor fluctuación turística atraída por el producto animación.

- Necesidad de una elevada comercialización y aceptación del producto animación por parte de los clientes.

Riesgos: La calidad de los servicios de animación y la preparación del personal no esté acorde con los objetivos trazados y con la imagen a ofrecer a los clientes.

\section{Prefactibilidad Organizacional:}

- Necesidad de una mayor preparación idiomática del personal y de un elevado sentido de pertenencia.

- Existencia de cuadros con prestigio dentro de la organización que pueden desarrollar con eficacia el proyecto.

- Capacitación del personal de animación de acuerdo a las necesidades actuales y futuras del centro.

Riesgos: Desmotivación del personal por contribuir a la mejora de la satisfacción del cliente.

- Pérdida de interés por la mejora continua.

\section{Prefactibilidad Medioambiental:}

- Hotel ubicado en una prestigiosa franja de playa (Sector de Los Taínos) y rodeado de una exuberante vegetación, hermosos jardines y espejos de agua.

Riesgos: Contaminación acústica y de estas principales áreas por el accionar inadecuado del personal y de los clientes.

\section{Prefactibilidad Técnica:}

- Dificultades con el equipo de audio.

- Insuficientes sillas en áreas recreativas.

- Carteleras de animación insuficientes.

- Pocos establecimientos para el ocio y la recreación. 
Riesgos: No disponer de los recursos materiales y humanos necesarios para efectuar con la calidad requerida el proceso de animación.

\section{Prefactibilidad Financiera:}

- Necesidad de realizar el proyecto en el mínimo tiempo posible, por los beneficios que representa una elevada satisfacción del cliente en el hotel, pues resulta rentable económicamente para la entidad ya que solucionaría problemas que potenciarían las ventas del hotel.

- Bajos ingresos por concepto de comercialización.

\section{Riesgos:}

- No disponer de un marco financiero inicial para el proyecto.

- Existencia de otros proyectos en la entidad que absorban el presupuesto de la entidad.

\section{Prefactibilidad Económica:}

- La tasa de interés para el financiamiento del proyecto es de $10 \%$.

- El presupuesto para la realización de la alternativa A es de $\$ 1840$ y para la alternativa B es de $\$ 1490$.

Después de haber realizado el análisis de los principales indicadores económicos se llega a la conclusión siguiente:

La Alternativa seleccionada es la $\mathrm{B}$, pues presenta una mayor rentabilidad económica y su período de recuperación es menor (aproximadamente de 5 meses).

\section{Matriz Riesgo - Niveles}

Después de realizada la matriz se concluyó que las prefactibilidades financieras y económicas presentan riesgos en la finalidad, objetivos y los resultados del proyecto riesgos, así mismo la organizacional tiene riesgos en este último. Además las prefactibilidades medioambientales y de mercado presentan riesgos en los resultados y la técnica en los insumos.

\section{Manejo de los riesgos:}

Para manejar los riesgos que pueden presentarse a la hora de realizar el proyecto se realizó la tabla 1 donde se exponen los diferentes tipos de riesgos, así como las consecuencias y las acciones a acometer en cada caso.

Tabla 1: Manejo de los riesgos del proyecto 


\begin{tabular}{|c|c|c|}
\hline $\begin{array}{ll}\text { Tipo de } \\
\text { riesgo }\end{array}$ & Consecuencias & Acciones a acometer \\
\hline Mercado & $\begin{array}{l}\text {-Destrucción de la imagen creada del } \\
\text { producto, provocando la obtención de } \\
\text { bajos ingresos por la poca aceptación de los } \\
\text { clientes. }\end{array}$ & $\begin{array}{l}\text {-Realizar encuestas de satisfacción } \\
\text { al cliente. } \\
\text {-Ofrecer cursos de atención a } \\
\text { clientes para el personal. }\end{array}$ \\
\hline $\begin{array}{l}\text { Organizaci } \\
\text { onal }\end{array}$ & $\begin{array}{l}\text { - No realización del proyecto a causa del } \\
\text { poco comprometimiento del personal } \\
\text { responsable del mismo. }\end{array}$ & $\begin{array}{l}\text {-Incentivar en el personal el } \\
\text { sentido de pertenencia. } \\
\text {-Efectuar charlas educativas sobre } \\
\text { la importancia de la mejora } \\
\text { continua y la necesidad de elevar } \\
\text { los índices de satisfacción del } \\
\text { cliente. }\end{array}$ \\
\hline $\begin{array}{l}\text { Medioamb } \\
\text { iental }\end{array}$ & $\begin{array}{lcr}\text {-Deterioro del } & \text { ecosistema } & \text { que rodea las } \\
\text { principales } & \text { áreas } & \text { recreativas, } \\
\text { desapareciendo } & \text { sus } & \text { atractivos } \\
\text { fundamentales. } & & \end{array}$ & $\begin{array}{l}\text {-Tratamiento adecuado de los } \\
\text { desechos resultantes. } \\
\text {-Mantener a un volumen adecuado } \\
\text { la música resultante de las } \\
\text { actividades realizadas. }\end{array}$ \\
\hline Técnica & $\begin{array}{l}\text {-Aumento considerable de la insatisfacción } \\
\text { de los clientes. } \\
\text {-No cumplimiento del proyecto en el } \\
\text { período estimado. }\end{array}$ & $\begin{array}{l}\text {-Evaluar la calidad de los } \\
\text { productos ofertados y las } \\
\text { competencias laborales del equipo } \\
\text { del proyecto. }\end{array}$ \\
\hline $\begin{array}{l}\text { Finan- } \\
\text { ciera }\end{array}$ & $\begin{array}{l}\text {-Incapacidad de realizar el proyecto de } \\
\text { mejora del índice de satisfacción del cliente } \\
\text { en el proceso de Animación. }\end{array}$ & $\begin{array}{l}\text {-Evaluar nuevas vías de } \\
\text { financiamiento para el proyecto. }\end{array}$ \\
\hline
\end{tabular}

Fuente: Elaboración propia.

\section{Lógica de Intervención}

Para ello se realizó la matriz de marco lógico donde se exponen los diferentes niveles del proyecto y los pasos y acciones a seguir en cada uno de ellos así como los indicadores a verificar, los medios para realizarlo y las condiciones críticas de ello.

\section{Estructuras de Organización}


La organización del personal asignado al proyecto será mediante la organización Monitor en la cual el director del proyecto no planifica ni decide, se limita a asesorar al director de la empresa, por lo que no tiene autoridad ni asume ninguna responsabilidad.

\section{Identificación del director de proyecto}

La persona responsable de la ejecución o supervisión del proyecto es el actual jefe de mantenimiento, puesto que en el hotel no existe un departamento de inversión. El mismo es graduado de Licenciatura en Economía, ha pasado varios entrenamientos en Venezuela y Cancún sobre gestión hotelera y tiene una elevada fluidez tanto en idioma Inglés, como en Francés e Italiano. Además, mantiene un elevado prestigio ante sus compañeros y un correcto porte y aspecto, teniendo todos los conocimientos y cumpliendo los requisitos necesarios para la dirección de este proyecto.

\section{Estructura de división del trabajo}

Para estructurar la división del trabajo se le asigna a cada miembro del equipo diversas actividades las cuales tienen un comienzo, un fin y recursos necesarios para su realización.

\section{Matriz de responsabilidades}

Para ello se les asignan diversas responsabilidades a los miembros del equipo en dependencia de su nivel de capacitación e implicación en el proyecto.

\section{Programación del proyecto}

Para realizar la programación del proyecto se utilizó el software Microsoft Project en donde se realizó el diagrama de Gantt y el de redes con los cuales se determinó la duración del proyecto tomando como base la programación de las actividades.

\section{Asignación de recursos}

Los recursos fueron asignados en dependencia de las actividades a realizar, la duración de las mismas y la cantidad de recursos a asignar.

\section{Control del costo}

Para el control de los costos se asignó un presupuesto dependiendo de las actividades a realizar en el proyecto.

\section{Conclusiones.}

- El análisis de la situación actual del producto animación en el hotel, permitió detectar las principales deficiencias del proceso, así como los criterios de los clientes. 
- El análisis de los problemas arrojó como problema fundamental el bajo índice de satisfacción del cliente en el proceso de animación.

- El análisis de los objetivos definió como objetivo fundamental del proyecto elevar el índice de satisfacción del cliente en el proceso de animación.

- Se proponen dos alternativas de proyectos para darle cumplimiento al objetivo definido.

- Se realiza el análisis de factibilidad, así como el análisis y manejo de los riesgos de las alternativas propuestas y se selecciona como la mejor alternativa: mejorar la profesionalidad de los animadores mediante cursos de capacitación, tanto en la comunicación idiomática como interpersonal para mejorar la forma de interactuar con los clientes.

- Se realizó la matriz de marco lógico donde se exponen los diferentes niveles del proyecto y los pasos y acciones a seguir en cada uno de ellos así como los indicadores a verificar, los medios para realizarlo y las condiciones críticas de ello.

- Se organizó el personal asignado al proyecto mediante la organización Monitor y se propone como director de proyecto un trabajador del hotel.

- La estructura de división del trabajo, la matriz de responsabilidades, la programación del proyecto, la asignación y nivelación de recursos y el control de los costos, permitieron planificar las actividades previstas a realizarse en el proyecto que se propone.

- Se realiza una propuesta de proyecto para lograr elevar el índice de satisfacción de los clientes en el proceso de animación en el hotel, para de esta forma lograr su fidelización con el producto y aumentar la comercialización.

\section{Referencias bibliográficas.}

OMT (2003). Turismo, conceptualización.

Padilla Rodríguez, N. (2015). Alianzas estratégicas entre prestadores de servicios turísticos estatales y no estatales, con enfoque de mercado. Tesis de Diploma en opción al título de Licenciado en Turismo. Universidad de Matanzas.

OMT (2015). Panorama OMT del turismo internacional. Edición 2015. Disponible en: www.e-unwto.org Consultado en noviembre de 2015.

Labollita, M. y Farré, M. (2005). Guía didáctica. Animación en el ámbito turístico. Ediciones Pirámide. España. ISBN: 84-368-1954-3.

Fernández, R. 2014. Propuesta de un programa de animación sociocultural para el Hotel Playa Girón. [Tesis de Diploma], en opción al título de Licenciado en Turismo. Departamento de Turismo. Universidad de Matanzas. Matanzas, Cuba. 

abril-junio, 2019

Ferreiro, I. 2010. La Comunicación Interna en el Proceso de Animación en el Hotel Sandals Royal Hicacos Resort \& SPA. [Tesis de Diploma ], en opción al título de Licenciado en Turismo Departamento de Turismo. Universidad de Matanzas. Matanzas, Cuba.

Montano, A. 2013. Propuesta del manual de procedimientos para la gestión de la Animación en el hotel Karey Punta Blanca. [Tesis de Diploma], en opción al título de Licenciado en Turismo. Departamento de Turismo. Universidad de Matanzas. Matanzas, Cuba.

Zamora Tabares, D. T. et al (2009). Gestión y evaluación de proyectos turísticos. Ciudad de La Habana.

Heredia Scasso, R. (1995). Dirección integrada de proyecto-DIP-Project Management. Segunda edición. España.

Polaino de los Santos, C. (). Introducción a la gestión de proyectos. CETDIR. Ciudad de la Habana.

Consejos de Ministros (2014). Decreto No. 327/2014: Reglamento del Proceso Inversionista. Gaceta Oficial No. 5 Extraordinaria 27 de enero de 2015. La Habana, Cuba.

Consejos de Ministros (2011). Lineamientos de la Política Económica y Social de Cuba. VI Congreso del Partido Comunista de Cuba. La Habana, Cuba.

Jiménez Valero, B. (2010). Selección de lecturas de dirección integrada a proyectos. Universidad de Matanzas, Cuba. 


\section{Para citar el artículo indexado}

Jiménez Valero, B., Lorenzo Puentes, M., \& Pino Acosta, L. (2020). Proyecto de mejora del índice de satisfacción del cliente en el proceso de Animación en el hotel Meliá Las Antillas de Varadero. AlfaPublicaciones, 1(1), 6-19. https://doi.org/10.33262/ap.v1i1.16

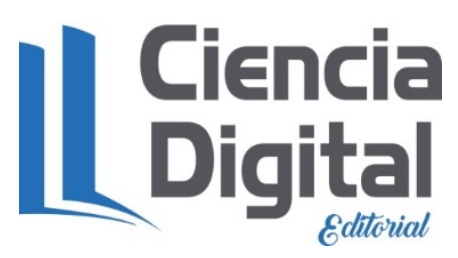

El artículo que se publica es de exclusiva responsabilidad de los autores y no necesariamente reflejan el pensamiento de la Revista Alpha Publicaciones.

El artículo queda en propiedad de la revista y, por tanto, su publicación parcial y/o total en otro medio tiene que ser autorizado por el director de la Revista Alpha Publicaciones.
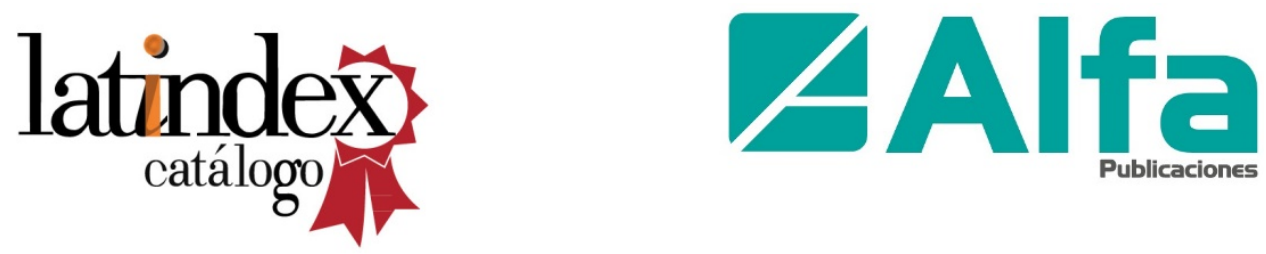